\title{
Pricing foreign equity option under stochastic volatility tempered stable Lévy processes
}

\author{
Xiaoli Gong* Xintian Zhuang \\ School of Business Administration, Northeastern University, Shenyang, 110169, China
}

\begin{abstract}
Considering that financial assets returns exhibit leptokurtosis, asymmetry properties as well as clustering and heteroskedasticity effect, this paper substitutes the logarithm normal jumps in Heston stochastic volatility model by the classical tempered stable (CTS) distribution and normal tempered stable (NTS) distribution to construct stochastic volatility tempered stable Lévy processes (TSSV) model. The TSSV model framework permits infinite activity jump behaviors of return dynamics and time varying volatility consistently observed in financial markets through subordinating tempered stable process to stochastic volatility process, capturing leptokurtosis, fat tailedness and asymmetry features of returns. By employing the analytical characteristic function and fast Fourier transform (FFT) technique, the formula for probability density function (PDF) of TSSV returns is derived, making the analytical formula for foreign equity option (FEO) pricing available. High frequency financial returns data are employed to verify the effectiveness of proposed models in reflecting the stylized facts of financial markets. Numerical analysis is performed to investigate the relationship between the corresponding parameters and the implied volatility of foreign equity option.
\end{abstract}

Keywords: stochastic volatility; tempered stable Lévy process; foreign equity option

\section{Introduction}

With the development of economic globalization and liberalization trade, foreign equity option related to currency transformation becomes more and more important for regulating multidimensional risks. As a contingent claim, its nominal payoff and actual payoff are done in two different currency forms respectively. Plentiful evidence has illustrated that returns distribution exhibits leptokurtosis, asymmetry and volatility clustering phenomena, leading to stochastic jumps in asset prices. The traditional financial asset dynamic model based on normal distribution cannot capture the asymmetric leptokurtic properties and volatility smile phenomenon. It has been demonstrated by Ait-Sahalia et al. [1] and Klingler et al. [2] that stochastic volatility and jumps are inherent nature of stock price dynamics in explaining stylized facts in financial markets. Therefore, when describing foreign equity dynamics and exchange rate price dynamics, it is reasonable to incorporate both stochastic volatility and leptokurtosis property in modeling. Considerable efforts have been made to relax these restrictions in pricing FEO option to accommodate abnormality returns and heteroskedasticity effect which leads to extensive alternative models. Huang and Hung [3] priced foreign equity option utilizing Lévy processes while assuming constant volatility. Ma [4] proposed a new stochastic volatility (SV) model to price FEO without jumps in foreign asset prices. Sun and Xu [5] priced FEO with SV models, giving analytical expression for logarithm normal jumps driven SV models that belong to finite activity jumps diffusion process. In this paper, we unify the pricing framework for tempered stable Lévy processes driven SV models that comprise finite jumps and infinite jumps and especially focus on the case where the Lévy jumps are infinite to capture both large and small jumps in stock returns.

The infinite activity tempered stable (TS) processes presented in Rosinski [6] act as an useful tool to capture the high peak and fat tail in asset returns have been validated superior to the $\alpha$ stable Paretian distribution which is insufficient to explain the dynamics of stock processes flexibly due to its infinite moments. It has been addressed in Carr and $\mathrm{Wu}$ [7] that infinite activity Lévy processes with converged moments can capture both consistently observed large jumps and small jumps in asset price dynamics. It generates infinite jumps within finite interval and consists of classical tempered stable distribution process in Carr et al. [8], normal tempered stable distribution process and rapidly decreasing tempered stable distribution process in [9] and so on. Noticeably, the CGMY distribution which is essentially equivalent to CTS distribution is extensively studied by Kuchler et al. [10] and Zaevski et al. [11]. The TS distributions are obtained through making $\alpha$ stable law multiply their corresponding Lévy measures generated from moment generating functions. It is equipped with more flexible ability in capturing asymmetry, higher kurtosis and fatter tails compared with other infinite activity Lévy processes such as the Variance Gamma process (VG) presented in Madan and Carr [12], Normal Inverse Gaussian process (NIG) presented in Barndorff Nielsen [13] when combined with stochastic volatility components [14]. Moreover, the infinite activity characteristic of TS processes can better describe the infinite jumps phenomenon exists in the underlying process $[15,16]$. Additionally, the availability of closed form characteristic functions of Lévy processes makes it possible for deriving density functions of these distributions employing fast Fourier transform technique so that foreign equity option can be calculated conveniently.

The principal approach to handle with heteroskedasticity and volatility clustering effect in returns variance is the application of stochastic volatility models [17-19]. Regarding the time varying volatility models with jumps,

\footnotetext{
* Corresponding author. Tel.: +86 2483679139 .

E-mail address: qdgongxiaoli@126.com
} 
Ouadghiri et al. [20] performed research on stochastic volatility models with jumps components in foreign exchange markets. Although vast researches have been performed on jump-diffusion stochastic volatility models [21-23], it is evident that infinite jumps are identifiable in foreign equity data and exchange rate data. It is more reasonable to substitute finite jump part to allow both infinite jumps and stochastic diffusion components in option pricing models. The same conclusion can be found in [7], who expressed that introducing Lévy models into permitted stochastic volatility processes generates excessive skews, kurtosis and persistent heteroskedasticity in underlying asset price process. Schoutens et al. [24] proposed the traditional Lévy driven stochastic volatility model framework employing time changed variance out of a Cox-Ingersoll-Ross (CIR) square root stochastic clock and it covered predominant Lévy driven stochastic processes such as VGCIR process and NIGCIR process and so on where it increased or decreased the uncertainty of economy through speeding up or slowing down the elapsed time. Zaevski et al. [11] turned attention to the stochastic volatility models with CTS Lévy jumps so that it can describe the leptokurtosis, asymmetry property in returns and varying volatility. In order to capture all these properties more efficiently, we enhance the prevailing models from time changed classical tempered stable distribution (CTSSV) to time changed normal tempered stable distribution (NTSSV) to reconstruct the time changed tempered stable model framework.

The improvement contribution of our work over the prevailing is that we subordinate normal tempered stable processes to time varying stochastic volatility processes to construct time changed tempered stable model framework so as to capture the distributional behavior of asset returns more effectively. By exploiting Fourier transform technique and characteristic function for probability calculation, the closed form solutions for foreign equity option is obtained.

\section{Stochastic volatility tempered stable Lévy processes for foreign equity option}

\subsection{CIR stochastic clock}

The stochastic variation in volatility can be featured by instantaneous time changes. The model employed to generate stochastic volatility is the integrated square root CIR process, which describes the instantaneous activity rate $v_{t}$ utilizing the following stochastic differential equation $d v_{t}=\kappa\left(\eta-v_{t}\right) d t+\lambda \sqrt{v_{t}} d W_{t}$, with the corresponding parameters $\kappa$ denoting the speed of mean reversion, $\eta$ representing the long term variance, $\lambda$ standing for the volatility of volatility, fulfilling $\kappa \eta>\lambda^{2} / 2$ to assure the non-negativity of instantaneous volatility. The CIR mean reverting process acts as measurement of time change rate through speeding up or slowing down the elapsed time according to the economic events so as to increase or decrease the activity intensity, thus capturing the volatility clustering and heteroskedasticity effect in foreign equity data and exchange rate data.

The reason for choosing the mean reverting CIR process to measure the time change rate in building instantaneous volatility model is that the economic time $T_{t}$ passed in $t$ units time can be computed by integral of $v_{t}$ between ranges from zero to time $t$, making time stochastic by the built in stochastic clock. The original clock can be regarded as calendar time and the new stochastic clock can be seen as business time, implying that faster business clock suggests more randomness in volatility and thus higher volatility for asset returns. We define the stochastic time change variable $T_{t}$ within $[0, t]$ as $T_{t}=\int_{0}^{t} v(\mathrm{~s}) d s$, and then the characteristic function of CIR can be computed in the following form.

$$
\varphi\left(u, t, y_{0}\right)=\frac{\exp \left(\frac{\kappa^{2} \eta t}{\lambda^{2}}\right)}{\cosh \left(\frac{\gamma t}{2}\right)+\frac{\kappa}{\gamma} \sinh \left(\frac{\gamma t}{2}\right)^{2 \kappa \eta / \lambda^{2}}} \exp \log ^{y_{0} \frac{2 i u}{\kappa+\operatorname{coth}\left(\frac{\gamma t}{2}\right) \gamma}}
$$

with $\gamma=\sqrt{\kappa^{2}-2 \lambda^{2} i u}$.

\subsection{Infinite activity tempered stable Lévy processes}

We generalize the Heston model in alternative models that allows infinite activity CTS jumps and NTS jumps in asset returns process to replace the Brownian motion process with tempered stable process. The underlying asset of FEO is foreign equity, the strike price can be chosen in domestic currency or foreign currency forms, whereas the payoff of option has to be transformed into domestic currency. In this paper we suppose the strike price $K_{D}$ in domestic form, which is reasonable for investors. Let $F_{t}, S_{t}$ respectively represent time varying exchange rate in domestic/foreign currency and asset price in foreign currency on expiration in market models, then $F_{t} S_{t}$ represents the underlying asset price of FEO. The FEO has the payoff expressed in form of FEO $=\left(F_{t} S_{t}-K_{D}\right)^{+}$, and can be specified as an exponential affine function of the tempered stable Lévy process $X_{t}, F_{t} S_{t}=F_{0} S_{0} \exp \left(X_{t}\right)$.We assume that $S_{t}$ and $v_{t}$ are independent, then we model the dynamic underlying assets of foreign equity employing TSSV 
process under risk neutral measure, it can be expressed as follows:

$$
\begin{gathered}
d S_{t}=\left(r_{d}-\bar{\mu}\right) S_{t} d t+\sqrt{v_{t}} d W_{t}+J_{t} d N_{t} \\
d v_{t}=\kappa\left(\eta-v_{t}\right) d t+\lambda \sqrt{v_{t}} d B_{t}
\end{gathered}
$$

where $J_{t}$ is chosen to be tempered stable processes following the CTS distribution and NTS distribution so as to account for sophisticated jump structures and market structure. Eq.(2) expresses the dynamic process of stock prices, Eq.(3) expresses the evolution of activity rate process and can be simulated in various discretization schemes. Certainly, $J_{t}$ can be specified as other distributional forms, for example, in [25-27], it is assumed to follow the double exponential distribution, generating double exponential jump diffusion stochastic volatility process. $W_{t}$ and $B_{t}$ respectively denote independent Wiener processes driven by different factors.

Let $\varphi(u)$ represent the characteristic function of infinite activity tempered stable Lévy processes, we define the cadlag Lévy process $\left(X_{t}\right)_{t \geqslant 0}$ in probability space $(\Omega, F, P)$ an infinite divisible distribution which has independent and stationary increments as well as $n$th power of characteristic functions $\varphi(u)$. The infinite activity Lévy distribution has a triplet of characteristics $(\mu, \sigma, v)$, where it denotes a linear deterministic component, a Brownian motion component and a pure jump part, respectively. Given that the distribution function forms for Lévy processes are generally complicated, we employ characteristic function expression $\varphi(u)=E\left[e^{i u X(t)}\right], u \in \mathrm{R}$, or characteristic exponent $\psi(u)=(-1 / t) \log \varphi(u), t \geqslant 0$, expression for convenience. The characteristic exponent $\psi(u)$ meets the following equation according to the Lévy-Khintchine formula:

$$
E[\exp (i u X)]=\exp \left(-i u o+\frac{\sigma^{2} u^{2}}{2}-\int_{-\infty}^{+\infty}\left(e^{i u x}-1-i u x_{|x| \leq 1}\right) v d x\right)
$$

where $o$ represents the drift that controls the location of distribution, $v$ refers to the Lévy measure which suggests the arrival rate of jumps.

We suppose the returns process to follow tempered stable distributions and particularly pay attention to two kinds of tempered stable distributions: the classical tempered stable distribution and the normal tempered stable distribution. Using characteristic functions, the cumulant $c_{n}$ of tempered stable processes can be calculated through $_{c_{n}}=\frac{1}{i^{n}} \frac{d^{n}}{d u^{n}} \log E\left[e^{i u X}\right]$.

Let $\alpha \in(0,1) \cup(1,2)$ to guarantee the finite quadratic variation, $C, \lambda_{+}$and $\lambda_{-}>0, m \in \mathrm{R}$. The characteristic function of CTS distribution is calculated as follows:

$$
\varphi_{C T S_{X_{t}}}(u)=\exp \left(t \mathrm{C}_{1} \Gamma(-\alpha)\left(\lambda_{-}-i u\right)^{\alpha}-\lambda_{-}^{\alpha}+\left(\lambda_{+}+i u\right)^{\alpha}-\lambda_{+}^{\alpha}\right)
$$

where $\Gamma$ is the gamma function, $\mathrm{C}_{1}, \lambda_{-}, \lambda_{+}, \alpha$ denote parameters in classical tempered stable distribution model respectively.

The scale parameter $\mathrm{C}_{1}$ in CTS model associates with kurtosis of the density function, with smaller $\mathrm{C}_{1}$ resulting to higher peak. While parameters $\lambda_{+}$and $\lambda_{\text {- }}$ in classical tempered stable distribution grasp the tails decay rate in positive and negative sides, respectively, with parameters $\lambda_{-}, \lambda_{+}, \alpha$ related to the tails weights. If $\lambda_{+}$disagrees with $\lambda_{-}$, it is asymmetric in jumps structure. When $\lambda_{+}>\lambda_{-}\left(\lambda_{-}>\lambda_{+}\right)$, the returns distribution is left (right) skewed, otherwise, it exhibits symmetry in returns.

It is known that Lévy measure $v$ obtained from the FFT of characteristic functions plays significant role in reflecting jump behaviors and jumps structure of random distributions in addition to reflecting the jump intensity differences for different processes. The Lévy measure is associated with probability density distribution of the corresponding process, with the distributional width of measure related to the peak of density function, and its distributional height associated with the tail of density function. It describes the arrival rate of jumps for $X$, satisfying condition $\int_{R}\left(1 \wedge x^{2}\right) v(d x)<\infty$. And the Lévy measure of CTS distribution can be written as

$$
v_{C T S}(d x)=\frac{\mathrm{C}_{1} e^{-\lambda_{+} x}}{x^{1+\alpha}} 1_{x>0}+\frac{\mathrm{C}_{1} e^{-\lambda_{|x| x \mid}}}{|x|^{1+\alpha}} 1_{x<0}
$$

Let $\mu \in \mathrm{R}, \theta \in(0,1), v, \sigma>0$, the characteristic functions of NTS distribution is calculated as follows:

$$
\varphi_{N T S_{X_{t}}}(u)=\exp \left(\frac{(1-\theta) t}{v \theta}\left(1-\left(1-\frac{\left(i u \mu-\frac{\sigma^{2} u^{2}}{2}\right) v}{1-\theta}\right)^{\theta}\right)\right)
$$

where $\mu, v, \sigma, \theta$ denote parameters in normal tempered stable distribution model respectively.

The parameter $\mu$ in normal tempered stable distribution determines the skewness of density function, $v$ controls the excessive kurtosis exceeding normal distribution, $\theta$ affects the shape of returns distribution. When specifying $\theta=1 / 2$, it gives rise to the case of Normal Inverse Gaussian model, while approximating to zero, it leads to the case of Variance Gamma model. What can be concluded from above is that higher kurtosis in time series 
corresponds to higher value of $\theta$.

Furthermore, the Lévy measure of NTS distribution can be written as

$$
v_{N T S}(d x)=\sqrt{\frac{2}{\pi}}\left(\frac{v}{1-\theta}\right)^{\theta-1} \frac{e^{\frac{\mu x}{\sigma^{2}}}|x|^{-\theta-\frac{1}{2}}}{\sigma \Gamma(1-\theta)}\left(\mu^{2}-\frac{2(\theta-1) \sigma^{2}}{v}\right)^{\frac{\theta}{2}+\frac{1}{4}} K_{\theta+\frac{1}{2}}\left(\sqrt{\frac{x^{2}}{\sigma^{2}}\left(\frac{\mu^{2}}{\sigma^{2}}-\frac{2(\theta-1)}{v}\right)}\right)
$$

where $K$ denotes the modified Bessel function of the third kind. $v_{C T S}(\mathrm{R})=v_{N T S}(\mathrm{R})=\infty$, indicating that the CTS and NTS processes are pure jumps processes and possess infinite activity property.

\subsection{Time changed tempered stable Lévy processes}

Then we introduce the tempered stable distributions into the CIR process to formulate time changed TS model process $Z_{t}$ with $Z_{t}=X_{Y_{t}}$. By subordinating the tempered stable distributions to the stochastic volatility process, the stochastic volatility tempered stable Lévy processes are constructed, which accommodates jumps and stochastic volatility simultaneously. When $X_{t}$ follows the NTS process, $Z_{t}$ is the time changed NTS process, with the same way to time changed CTS process. Following [24], it is known that the characteristic function of $Z_{t}$ can be calculated as follows:

$$
\varphi_{Z_{t}}(u, t)=E\left[\mathrm{e}^{\mathrm{i} u Z_{t}}\right]=\varphi_{Y_{t}}\left(-\mathrm{i} \psi_{X_{t}}(u), t\right)=\mathcal{L}_{Y_{t}}\left(\psi_{X_{t}}(u)\right)
$$

where $\psi(u)$ denotes the characteristic exponent. And the characteristic function of $Z_{t}$ is converted into the Laplace transform of $Y_{t}$ at characteristic exponent of $X_{t}$.

The advantage of stochastic analysis method to characterize stochastic volatility over regime switching method and time series method is that it can be effectively applied in FFT because of the availability of analytical characteristic function form. In this paper, we introduce the stochastic time changes into the tempered stable processes framework by letting the variance of classical tempered stable distribution and normal tempered stable distribution change with time. This method had been used in Carr et al. [8] for time changed Lévy processes in European option pricing covering VGCIR and NIGCIR models. We extend its framework via incorporating normal tempered stable distribution with random time change to capture the information content of markets.

The pure jump infinite activity Lévy process driven stochastic volatility model consists of two parts: one part is a pure jumps Lévy process that controls asset price dynamic evolution process, the other part is a stochastic volatility process that controls the strength of price fluctuations and jump frequency by adjusting the time change rate. The foreign equity option uses foreign equities as underlying assets, with the strike prices generally being expressed in domestic currency and the payoff being converted into domestic currency. The payoff of this option can be expressed as FEO $=\left(F_{t} S_{t}-K_{D}\right)^{+}$, where $K_{D}$ denotes domestic form strike currency. Let $X_{t}$ represents the stochastic volatility tempered stable Lévy processes, then $F_{t} S_{t}$ becomes the underlying asset price of foreign exchange option, and the dynamic process of $F_{t} S_{t}$ can be given by $F_{t} S_{t}=F_{0} S_{0} \exp \left(X_{t}\right)$. The TSSV process is established through subordinating Lévy model to stochastic volatility process in order to reflect the jump behaviors and stochastic volatility of asset returns $\ln \left(F_{t} S_{t} / F_{0} S_{0}\right)$ simultaneously. It not only overcomes shortcomings of pure jump Lévy models that cannot reflect the diffusion process of underlying volatility, but also overcomes the defects of stochastic volatility models that cannot depict infinite jumping behaviors of returns. Then the characteristic function formula of logarithmic returns for TSSV model is derived analytically as follows:

$$
\begin{aligned}
e^{i u \ln \frac{F_{F} S_{t}}{F_{0} S_{0}}} & =\exp \left\{i u r t+\frac{2 \psi(u) Y_{0}\left(1-e^{-\Pi T}\right)}{2 \Pi-(\Pi-\kappa)\left(1-e^{-\Pi T}\right)}-\frac{k \eta}{\lambda^{2}}\left[2 \ln \left[1-\frac{\Pi-\kappa}{2 \Pi}\left(1-e^{-\Pi T}\right)\right]+(\Pi-\kappa) t\right]\right\} \\
& =\exp [\text { iurt }+\tau(t, u)]
\end{aligned}
$$

where $\Pi=\sqrt{\kappa^{2}-2 \psi(u) \sigma^{2}}, \psi(u)$ refers to the characteristic exponent of pure jump Lévy processes, $\kappa, \eta, \lambda$ are parameters of CIR stochastic volatility process. For CTSSV model, it contains seven parameters which include $C_{1}$, $\lambda_{+}, \lambda_{-}, \alpha, \kappa, \eta, \lambda$. For NTSSV model, it also consists of seven parameters which contain $\mu, v, \sigma, \theta, \kappa, \eta, \lambda$.

Then the probability density function can be obtained by fast Fourier transform of characteristic functions. In Fig. 1 we show the effects of the four parameters of CTS marginal distribution in stochastic volatility classical tempered stable model have on density function distribution of CTSSV model, and in Fig. 2 we show the influences of four parameters of NTS marginal distribution in stochastic volatility normal tempered stable model have on the density function distribution of NTSSV model. What can be seen is that parameters $\mathrm{C}_{1}, \alpha$ have inverse relationship with the peaks sharpness of CTSSV model density function, while $\lambda_{ \pm}$affect the overall position of density distribution in CTSSV model, $\theta$ and $v$ are oppositely correlated with the sharpness of the peaks in NTSSV model's density function, $\mu$ influences the overall position of NTSSV density distribution, and $\sigma$ affects the shape of the density function.

The introduction of infinite jump behaviors for underlying asset returns that are modeled by infinite activity Lévy processes can capture asymmetry, leptokurtosis and thicker tail properties in returns in addition to 
characterizing persistence effect and heteroskedasticity effect in volatility. The Lévy processes driven stochastic volatility models incorporates jumps and stochastic volatility simultaneously, which capture not only jumps but also stochastic volatility in stock price dynamics.
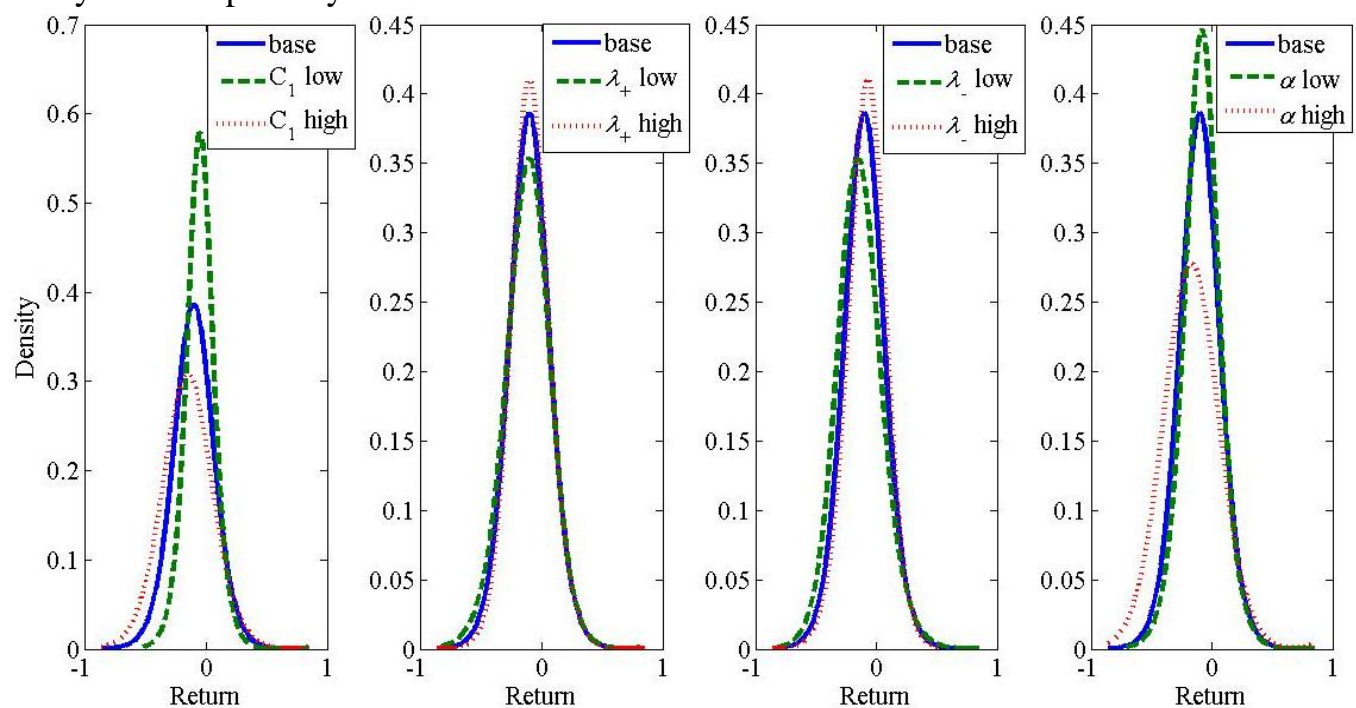

Fig.1. Probability density distribution of CTSSV model
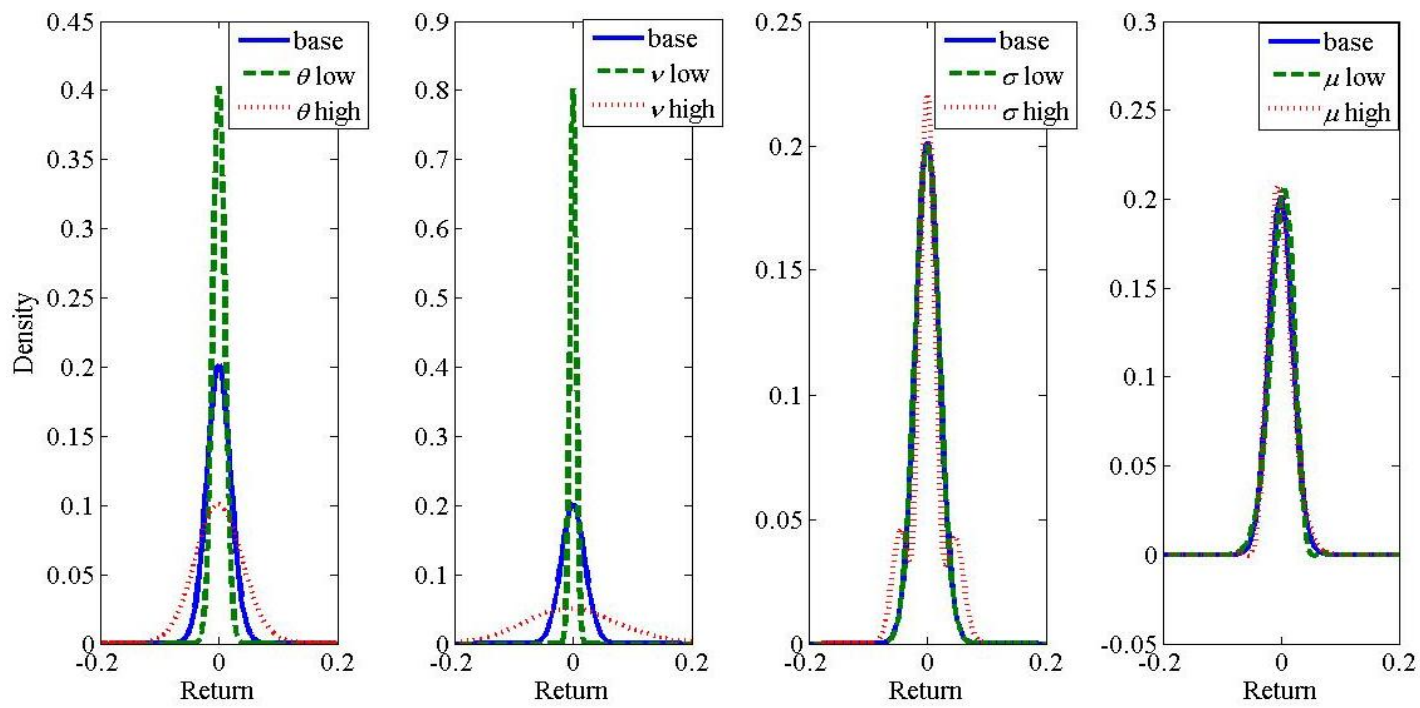

Fig.2. Probability density distribution of NTSSV model

\section{FFT and PDF for FEO stochastic volatility tempered stable Lévy processes}

Let $s_{t}$ denotes the logarithm of foreign equity price returns, $k=\ln K / F_{0} S_{0}$, the value of the foreign equity option $C(k)$ at initial time is represented as $C(k)=\mathrm{e}^{-r_{d} t} E^{Q}\left(\mathrm{e}^{s_{t}}-\mathrm{e}^{k}\right) 1_{s_{1} \geq k}$. We utilized the conversion of pricing formula suggested by Carr and Madan [28] using Fast Fourier Transform technique and can be applied conveniently if characteristic functions of the risk neutral asset price process is available. Because the integrand in direct integration is not square integrable, it entails the Fourier transform of the dampened FEO option price and the formula of FEO option can be expressed as

$$
c(k, T)=\frac{\mathrm{e}^{-\gamma k}}{\pi} \int_{0}^{+\infty} \mathrm{e}^{-i u k} \vartheta(u) d u,
$$

where $\gamma$ is a dampening parameter and $\vartheta(u)=\frac{\mathrm{e}^{-r T} \phi(u-(\gamma+1) i)}{\gamma^{2}+\gamma-u^{2}+i(2 \gamma+1) u}$.

A sufficient integrated condition for $c(k, T)$ requires $\vartheta(0)$ being finite, namely, 


$$
\vartheta(0)=\frac{\phi(-(\gamma+1) i)}{\gamma^{2}+\gamma}<\infty .
$$

It will generate a series of $\gamma:\left(\gamma_{+}, \gamma_{-}\right)$. Let $\gamma_{\operatorname{Max}}=\gamma_{+}-1, \gamma_{\operatorname{Min}}=\gamma_{-}-1$, the range $\left[\gamma_{\operatorname{Max}}, \gamma_{\operatorname{Min}}\right]$ contains all allowed $\gamma$. According to Lord et al. [29], we can choose $\gamma$ such that

$$
\gamma=\arg \min \left[-\gamma k+\ln \phi_{F}\left(t_{0}, T,-(\gamma+1) i\right)\right] .
$$

The fast Fourier Transform method can be employed to speed up the calculation. The FFT based technique has preponderance of simultaneously computing for all strikes. The FFT returns $N$ values of $k$ and for a regular spacing size of $h$ where the number of grid points $N$ is a power of value 2, the value for $k_{u}$ is $k_{u}=-b+h(a-1)$, with $a$ being an positive integer and $b=N h / 2$, giving us log strike levels ranging from $-b$ to $b$. The integral in (14) is approximated utilizing the Trapezoid rule and yields

$$
c\left(k_{u}\right) \approx \frac{\mathrm{e}^{-\gamma k_{u}}}{\pi} \sum_{j=0}^{N} \exp (-i z v(j-1)(a-1)+i b v(j-1)) \vartheta(v(j-1)) v .
$$

During the application of FFT, note that $v h=2 \pi / N$, let $u_{j}=v(j-1)$, with Simpson's weighting rule, the formula of foreign equity option price is

$$
c\left(k_{u}\right) \approx \frac{\mathrm{e}^{-\gamma k_{u}}}{\pi} \sum_{j=1}^{N} \exp \left(-i \frac{2 \pi}{N}(j-1)(a-1)+i b u_{j}\right) \vartheta\left(u_{j}\right) \frac{v}{3}\left[3+(-1)^{j}-\ell_{j-1}\right],
$$

where $\ell_{n}$ is the Kronecker delta function which is unity for $n=0$ and zero otherwise.

By utilizing the fast Fourier transform of the corresponding characteristic functions of time changed tempered stable Lévy processes, analytical expressions of their probability density functions are derived in Menn and Rachev [30] for $\alpha$ stable distribution, which overcomes the drawbacks of stable distribution that cannot be used for density calculation, and this method is extended to classical tempered stable distribution in Scherer et al. [31]. The availability of closed form characteristic functions of TSSV processes facilitate the application for this class process in pricing foreign equity option. The fast Fourier Transform technique can be utilized to expedite the calculation speed. Hence, the PDF, CDF can be derived efficiently utilizing fast Fourier transform method.

The PDF $f_{X}$ of random variable $X$ in the TSSV process is calculated from the characteristic function $\varphi(u)$ exploiting the inverse transform of Fourier transform as long as the expression of $\varphi(u)$ is known.

$$
f_{X}(x)=\frac{1}{2 \pi} \int_{-\infty}^{+\infty} e^{-i u x} \varphi_{X}(u) d x
$$

It is well known that Discrete Fourier transform (DFT) approximate the Fourier transform discretely mapping from vector $\left(x_{1}, \ldots, x_{N}\right)$ to vector $\left(y_{1}, \ldots, y_{N}\right)$ in the following form

$$
x_{j}=\sum_{k=1}^{N} y_{k} e^{-i \frac{2 \pi(j-1)(k-1)}{N}}, j=1, \ldots, N
$$

The fast Fourier transform can efficiently implement the DFT algorithm, and it approximates the PDF at point $x_{j}$ as follows

$$
\begin{aligned}
f_{X}\left(x_{j}\right) & \approx \frac{1}{2 \pi} \int_{-a}^{+a} e^{-i u x_{j}} \varphi(u) d u \\
& \approx \frac{a}{N}(-1)^{j-1} i e^{i \frac{\pi(j-1)}{N}} \sum_{k=1}^{N}(-1)^{k-1} \varphi\left(u_{k}^{*}\right) e^{-i \frac{2 \pi(j-1)(k-1)}{N}} \\
& =\frac{a}{N}(-1)^{j-1} i e^{i \frac{\pi(j-1)}{N}} \operatorname{FFT}_{j}\left(\left((-1)^{k-1} \varphi\left(u_{k}^{*}\right)\right)_{k=1, \ldots N}\right)
\end{aligned}
$$

where $\alpha \in \mathrm{R}$, denotes the integration limits of Fourier transform, $j$ and $k \in\left\{1, \ldots, N=2^{q}\right\}, q \in N$, influences the number of integration steps, $u_{k}=-a+(2 a / N)(k-1), u_{k}^{*}=\left(u_{k+1}+u_{k}\right) / 2, x_{j}=-N \pi / 2 a+(\pi / a)(j-1)$.

The DFT algorithm divides the integration space into equidistant interval of size $2 a / N$, and utilize middle point rule for approximation so as to minimize errors. It can be seen that the choice of $N$ and $a$ is so significant that parameter $a$ affects the sampling distance and interpolation interval, and $N$ influences the computational complexity. A reasonable choice of is a compromise between sampling errors of interpolation error and discretization error. The property of approximation is determined by the integrand function in each interval. The FFT technique is an $O$ $(N \ln N)$ algorithm for computing DFT in $x_{j}$.

It has been illustrated that the CDF $F_{X}=P(X<x)$ of random variable $X$ in the TSSV process can be computed by the following equation with $\rho>0$.

Then, it holds that

$$
F_{X}(x)=\frac{e^{\rho x}}{\pi} \mathfrak{R}\left\{\int_{0}^{\infty} e^{-i u x} \frac{\varphi_{X}(u+i \rho)}{\rho-i u} d u\right\}
$$




$$
\int_{0}^{\infty} e^{-i u x} \frac{\varphi_{X}(u+i \rho)}{\rho-i u} d u \approx \sum_{k=1}^{N} e^{-i u_{k}^{*} x_{j}} \frac{\varphi_{X}\left(u_{k}^{*}+i \rho\right)}{\rho-i u_{k}^{*}} \frac{2 a}{N}
$$
follows:

Utilize the calculation equation $x_{j} u_{k}{ }^{*}=-\pi(2 k-1) / 2+(2 \pi / N)((2 k-1) / 2)(j-1)$, the CDF $F_{X}$ can be expressed as

$$
F_{X}(x)=\frac{e^{\rho x}}{\pi} \mathfrak{R}\left\{\frac{2 a}{N} e^{-i \frac{\pi}{N}(j-1)} \operatorname{FFT}_{j}\left(\left((-1)^{k-1} i \frac{\varphi_{X}\left(u_{k}^{*}+i \rho\right)}{\rho-i u_{k}^{*}}\right)_{k=1, \ldots N}\right\}\right.
$$

\section{PDF fitting with stochastic volatility tempered stable Lévy processes}

In this section we fit the probability density function of the stochastic volatility tempered stable Lévy processes to the real financial market high frequency returns data to test the stylized fact of financial data with our proposed model framework comparing with celebrated Heston model. The reason for choosing high frequency returns data is due to its fine properties in containing efficient intraday information and can efficiently amplify the abnormal distribution properties of returns. We investigate the 5 minute data of Chinese Shanghai-Shenzhen 300 index returns (HS300) and American Standard \& Poor 500 Index (SP500) from wind database ranging from 2015. 1.1 to 2016.1 .1 .

For the considered distributions, maximum likelihood estimation method is utilized in fitting the time series data. Then the fitted parameters of CTSSV model are $C_{1}=6.2318, \lambda_{+}=28.9574, \lambda_{-}=16.5726, \alpha=0.7561, \kappa=6.3512$, $\eta=0.4597, \lambda=0.9264$ and $C_{1}=5.2569, \lambda_{+}=31.9163, \lambda=19.7804, \alpha=0.5284, \kappa=8.3155, \eta=0.3866, \lambda=0.7415$ in Chinese and American market, respectively, and the fitted parameters of NTSSV model are $\mu=-0.0705, v=15.0979$, $\sigma=0.0652, \theta=0.6360, \kappa=7.6702, \eta=0.5631, \lambda=0.8493$ and $\mu=-0.0523, v=17.1842, \sigma=0.0449, \theta=0.8279, \kappa=5.4821$, $\eta=0.3882, \lambda=0.9566$, in Chinese and American market, respectively. From the estimation results, we can find some common findings in both Chinese and American financial markets. The consequences of CTSSV model with $\lambda_{+}>\lambda_{\text {- }}$ illustrates the returns distribution in both financial markets are left skewed. The values of $\mu$ in NTSSV model are below zero, validating that the density distribution of HS300 index and SP500 index are left skewed. When it comes to the volatility diffusion components, the values of volatility reverting rate $\kappa$, variance of volatility $\eta$ in NTSSV models are lower than that in CTSSV model, this is probably because the strong power of normal tempered stable processes in capturing stylized facts decreases the persistence of the volatility diffusion. Fig. 3 and Fig.4 exhibit the fitted densities for the traditional Black-School model, Heston model, CTSSV model, NTSSV model and the empirical density of HS300 stock index returns and SP500 index returns, respectively, in which it displays clearly the leptokurtosis and fat tailedness of empirical returns. It can be illustrated from both figures that the NTSSV model performs better than other infinite activity jump models and finite jump model at the peak. And it is closer to real facts of stock index in different markets, reflecting the distributional properties of capital markets more accurately.

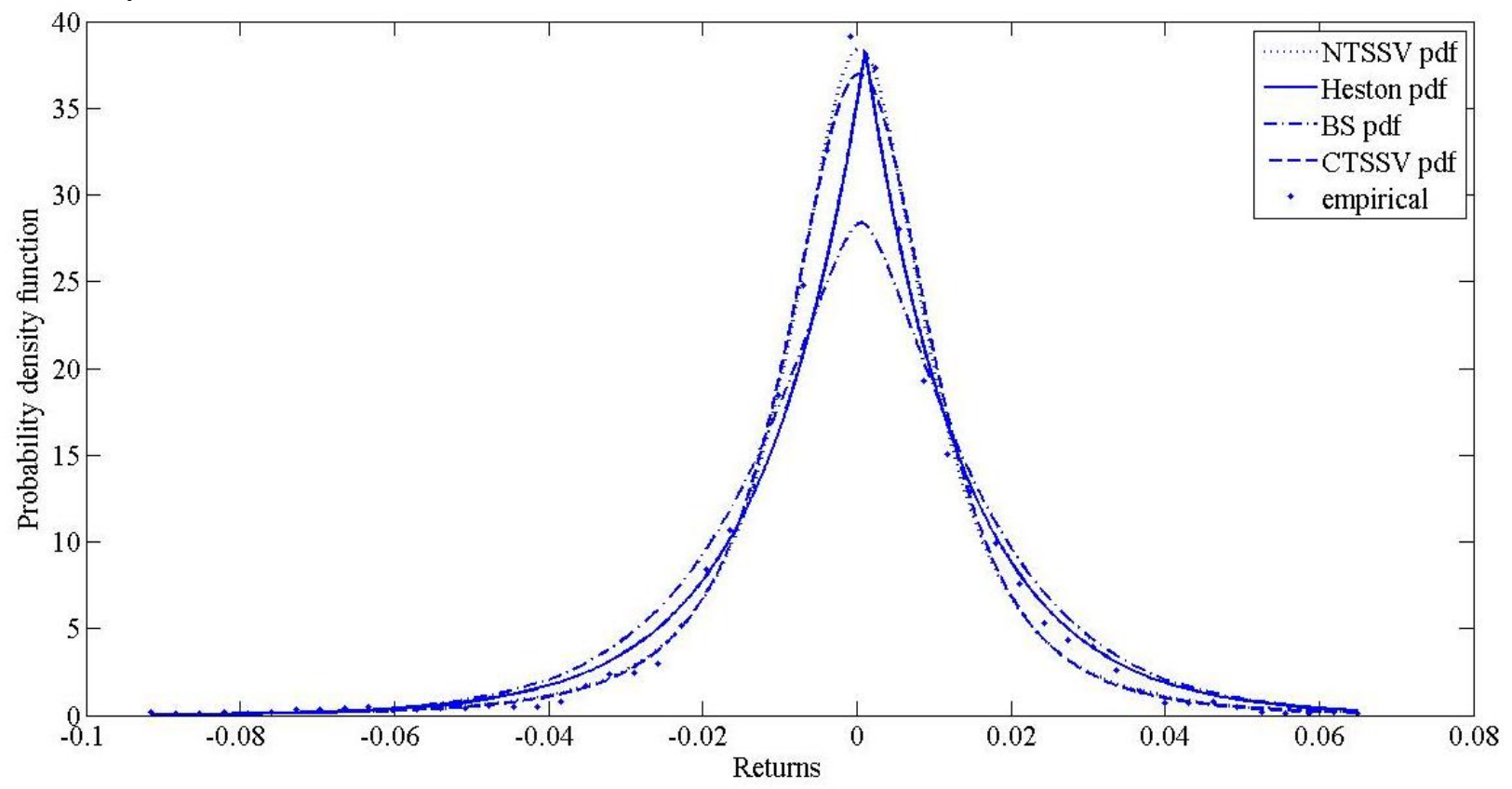

Fig. 3. Density distributions for HS300 stock index in different models 


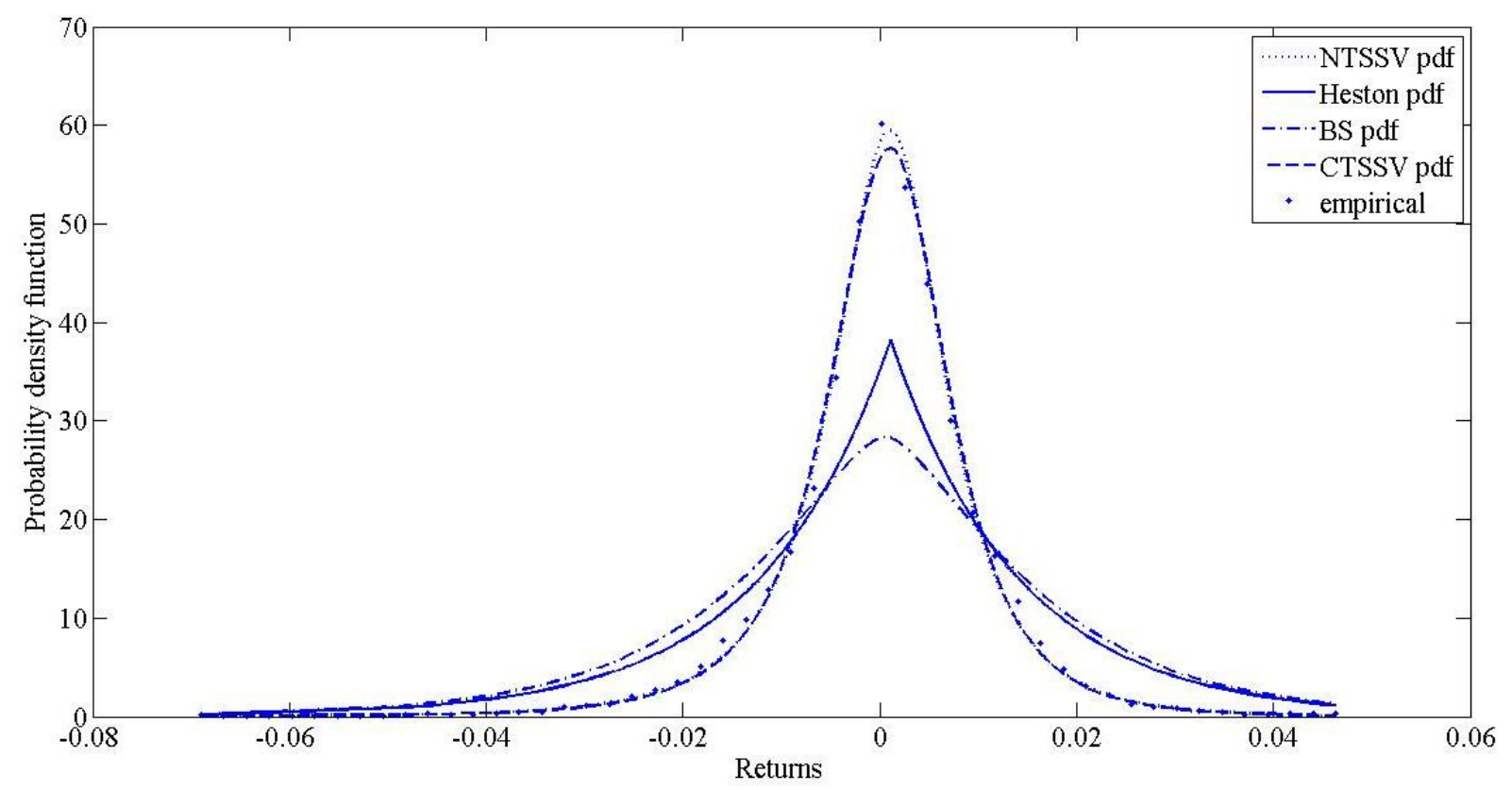

Fig. 4. Density distributions for SP500 stock index in different models

\section{Numerical Analysis}

Since there has no real foreign equity option in Chinese financial markets, we conduct numerical analysis to analyze the effects of correlated parameters in TSSV models on foreign equity option, which has exclusive correspondence relationship with implied volatility of option. The implied volatility of FEO option is used alternatively, with the investigated parameters containing the volatility of volatility $\lambda$, mean reverting rate $\kappa$ in CIR structures, marginal parameters of $C_{1}, \lambda_{+}, \alpha$ in CTSSV model, marginal parameters of $v, \sigma, \theta$ in NTSSV model. The initial values are set as follows $r_{d}=0.03, F_{0}=1, S_{0}=22, K_{D}=10, \ldots, 50, \mathrm{~T}=0.5, \ldots, 5$, and the comparative values set are listed in Table 1. We choose the initial values according to the empirical experiences in section 4 and those in [32].
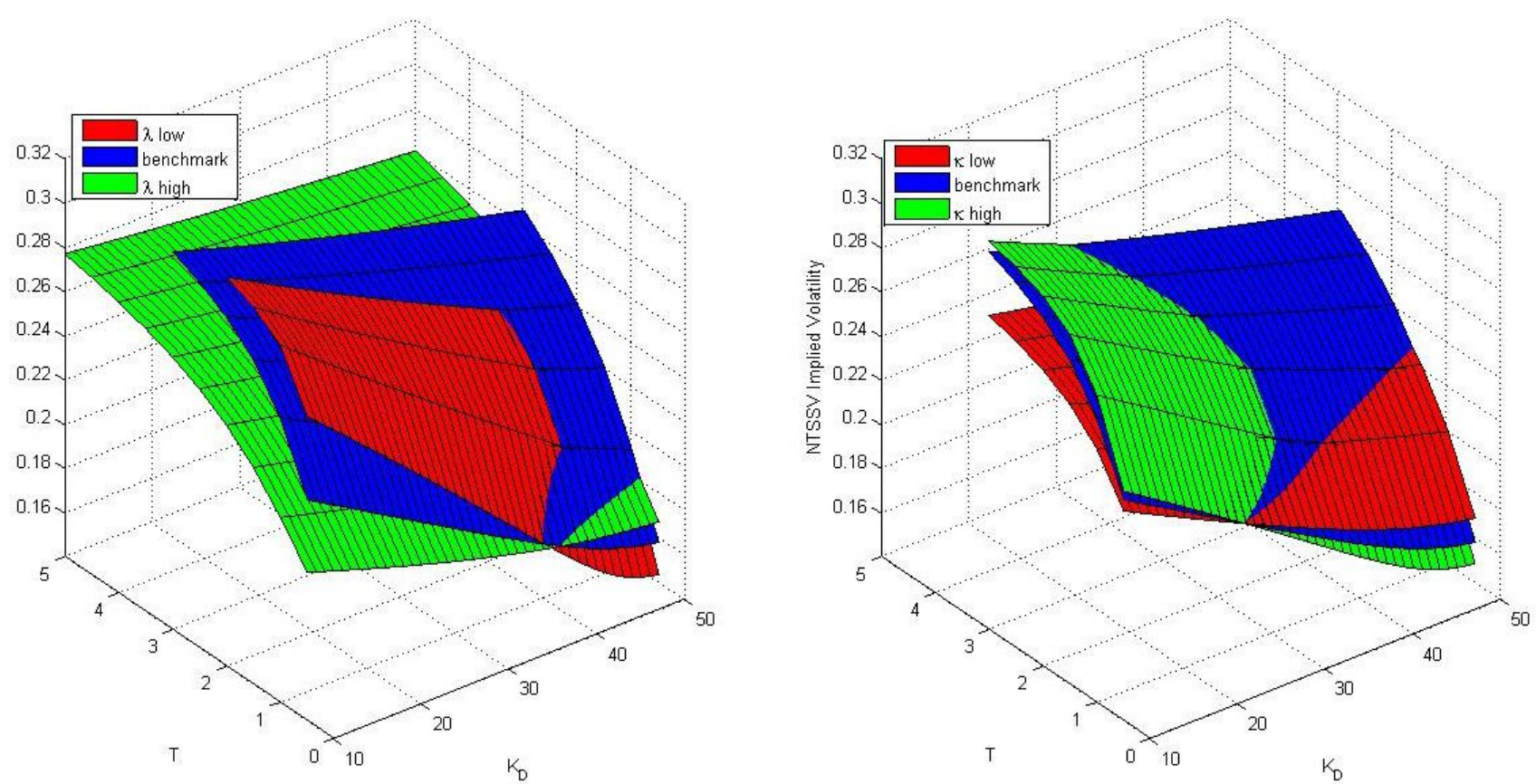

Fig. 5. The impact of $\lambda$ and $\kappa$ on FEO option implied volatility

Table 1 Comparative parameter values set 


\begin{tabular}{ccc}
\hline & CTSSV model & NTSSV model \\
\hline Low & $C_{1}=5, \lambda_{+}=25, \lambda=16, \alpha=0.5, \kappa=7, \eta=0.5, \lambda=0.8$ & $\mu=-0.07, v=12, \sigma=0.04, \theta=0.4, \kappa=6, \eta=0.5, \lambda=0.5$ \\
Benchmark & $C_{1}=6, \lambda_{+}=28, \lambda=16, \alpha=0.75, \kappa=7, \eta=0.5, \lambda=0.8$ & $\mu=-0.07, v=15, \sigma=0.06, \theta=0.6, \kappa=7, \eta=0.5, \lambda=0.8$ \\
High & $C_{1}=7, \lambda_{+}=30, \lambda=16, \alpha=0.9, \kappa=7, \eta=0.5, \lambda=0.8$ & $\mu=-0.07, v=18, \sigma=0.08, \theta=0.8, \kappa=8, \eta=0.5, \lambda=1.1$ \\
\hline
\end{tabular}
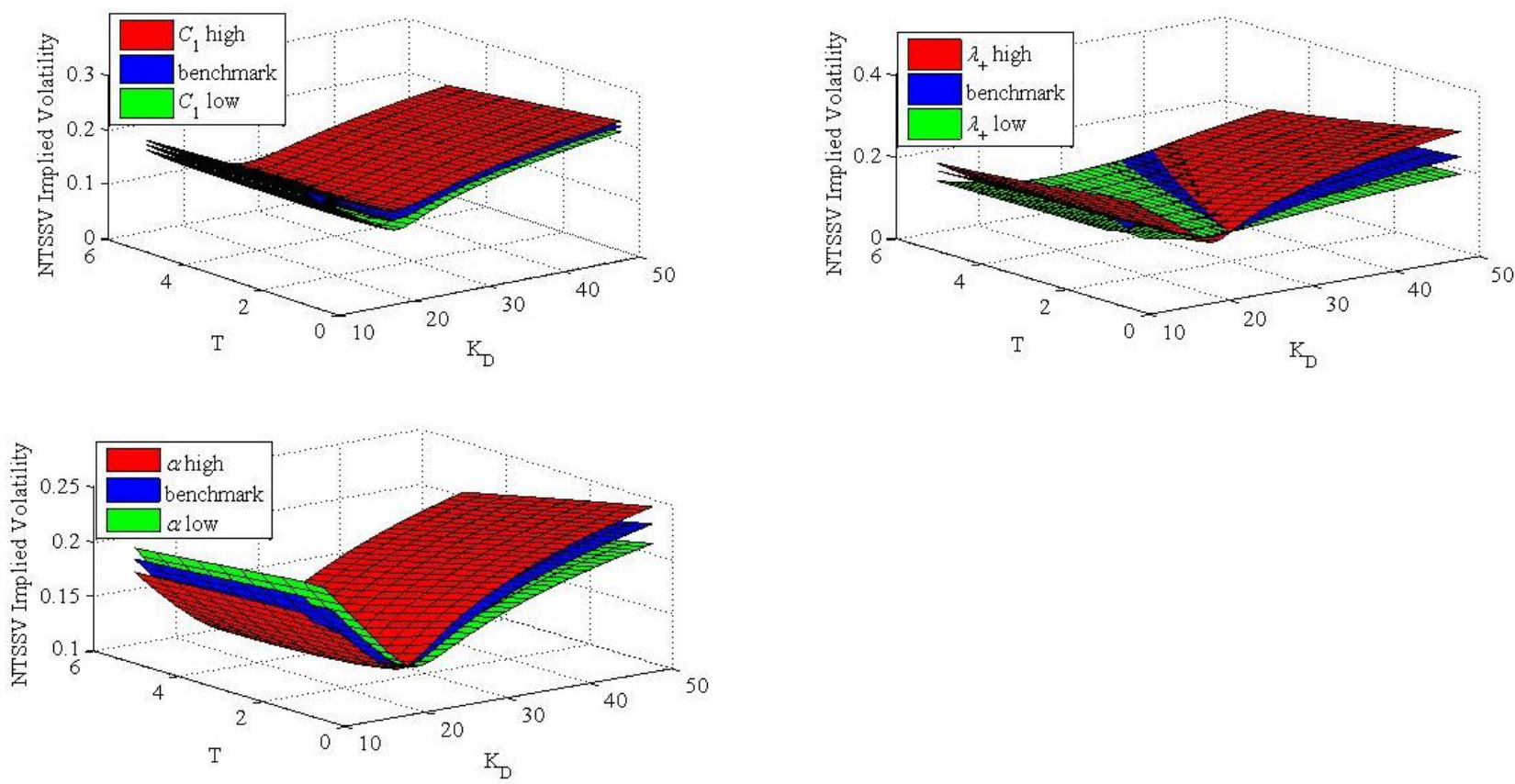

Fig.6. The impact of $C_{1}, \lambda_{+}, \alpha$ on FEO option implied volatility
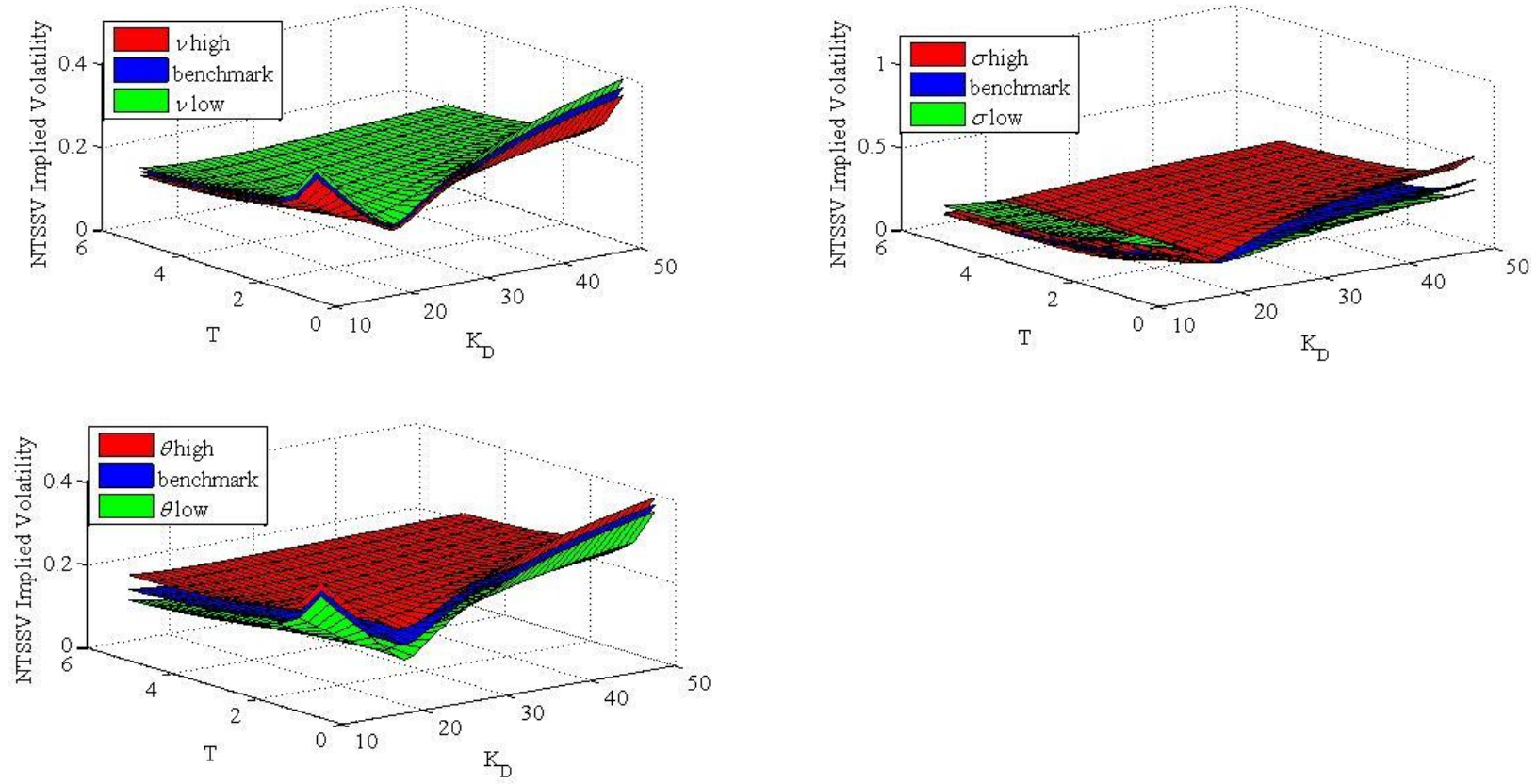

Fig.7. The impact of $v, \sigma, \theta$ on FEO option implied volatility

Fig.5 illustrates the relationships between FEO option implied volatility and the mean reversion rate $\kappa$, volatility of volatility $\lambda$ of NTSSV model. In this model, the variance from foreign equity drifts toward the long run mean $\eta$, while the mean reversion speed is controlled by $\kappa$. It can be inferred that higher average variance that produces higher variance $v_{t}$ leads to larger option prices. Fig. 1 displays that FEO implied volatility increases with larger $\kappa$, while it decreases with larger $\lambda$. The sensitivity of FEO implied volatility to volatility of volatility $\lambda$ is 
higher than that to mean reversion rate $\kappa$. As it can be seen from NTSSV model, parameter $\lambda$ increases the kurtosis of asset returns and creates fatted tails in tail distributions, lowering the market expectation of investors.

Fig. 6 shows the FEO implied volatility with different parameters of $C_{1}, \lambda_{+}, \alpha$ in CTSSV model, displaying the impacts of parameters $C_{1}, \lambda_{+}, \alpha$ on FEO option. It is evident that parameters $\alpha$ and $\lambda_{+}$that grasp the tails decay rate have different performance properties along with strike prices. We can observe that larger FEO implied volatility is obtained with the increase of $\lambda_{+}$in out-of-money option and in-the-money option, and the contrary correlation appears in at-the-money options. In our parameters setting, leptokurtic feature of foreign equity returns is well captured. It is remarkable that the sensitivity of parameter $\alpha$ with respect to FEO implied volatility displays complete reverse trends at both sides of strike. What can also be seen from Fig. 6 is that FEO implied volatility increases along with larger values of parameter $C_{1}$ that associates with kurtosis of asset returns.

Fig. 7 shows the FEO implied volatility under different parameters of $v, \sigma, \theta$ in NTSSV model, displaying the impacts of parameters $v, \sigma, \theta$ on FEO option. It can be seen that FEO implied volatility is an increasing function of $\sigma$ and $\theta$, while it is a decreasing function of values of $v$, which controls the excessive kurtosis exceeding normal distribution. Noticeably, when considering different strike prices, there is a remarkable volatility smiles appearing in at-the-money options. In addition, there is no significant difference between long maturities beyond three years. It can be inferred from the numerical analysis that the TSSV model exhibits effectiveness in the valuation of foreign equity option, the FFT technique is proved to be efficient.

\section{Conclusion}

The Gaussian distribution assumptions of assets returns have been reasonably rejected by empirical facts since the observed leptokurtosis, asymmetry property, as well as clustering and heteroskedasticity effect. This paper employs the infinite activity tempered stable Lévy processes and CIR model to construct stochastic volatility tempered stable models, which extend the Heston model by substituting logarithm normal jumps into normal tempered stable jumps to identify the distributional features in returns and volatility. The newly constructed model accommodates stochastic time that produces clustering volatilities and enhances previous models effectively in accounting for heavy tails and asymmetry jumps through the introduction of tempered stable distribution. By utilizing FFT technique, the foreign equity option is derived whose payoff is decided by equities in one currency but is accomplished in another currency. It entails the consideration of TSSV process description in both foreign equity price process and exchange rate process. Under the TSSV framework, analytical pricing formula is obtained by utilizing characteristic function. Empirical tests on high frequency financial returns validate the superiority of NTSSV model in capturing the leptokurtic and asymmetric properties of financial data. Numerical analysis is performed to further investigate the correlations between corresponding parameters and FEO implied volatility.

\section{Acknowledgments}

We sincerely thank our anonymous referee. We also would like to acknowledge the financial support from the National Science Foundation of China (Nos. 71571038, 71671030).

\section{References}

[1] Y. Ait-Sahalia, J. Jacod, J. Li, Testing for jumps in noisy high frequency data, J. Econ.168 (2) (2012) 207-222.

[2] S. Klingler, Y. S. Kim, T. Svetlozar, Option pricing with time-changed Lévy processes, Appl. Financ. Econ. 23 (15) (2013) 1231-1238.

[3] S.C. Huang, M.W. Hung, Pricing foreign equity options under lévy processes, J. Futures Markets 25 (2005) 917-944.

[4] J. Ma, Pricing foreign equity options with stochastic correlation and volatility, Ann. Econ. Finance 10 (2009) 303-327.

[5] Q. Sun, W. D. Xu, Pricing foreign equity option with stochastic volatility, Physica A 437 (2015) 89-100.

[6] J. Rosinski, Tempering stable processes, Stoch. Proc. Appl. 117 (6) (2007) 677-707.

[7] P. Carr, L. Wu, Time-changed Lévy processes and option pricing, J. Financ. Econ. 71 (2004) 113-141.

[8] P. Carr, H. Geman, D. B. Madan, M. Yor, The fine structure of asset returns: An empirical investigation, J. Business. 75 (2002) $205-332$.

[9] Y. S. Kim, S. Rachev, M. Bianchi, Time series analysis for financial market meltdowns, J. Bank. Finance 35 (2011) $1879-1891$.

[10] U. Kuchler, S. Tappe, Tempered stable distributions and processes, Stoch. Process Appl. 123 (2013) 4256-4293.

[11] T. S. Zaevski, Y. S. Kim, F. J. Fabozzi, Option pricing under stochastic volatility and tempered stable Lévy jumps, Int. Rev. Financ. Anal. 31 (2014) 101-108.

[12] D. Madan, P. Carr, E. Chang, The variance gamma process and option pricing, Eur. Financ. Rev. 2 (1998) $79-105$.

[13] O. E. Barndorff-Nielsen, Processes of normal inverse Gaussian type, Financ. Stoch. 2 (1997) 41-68.

[14] X.L. Gong, X.T. Zhuang, Option pricing for stochastic volatility model with infinite activity Lévy jumps, Phys. A. 455 (2016) $1-10$.

[15] J. Gajda, A.Wyłomańska, Tempered stable Lévy motion driven by stable subordinator, Phys. A. 392 (2013) 3168-3176.

[16] X.F. Ruan, Equilibrium asset pricing under the Lévy process with stochastic volatility and moment risk premiums, Econ. Model. 54 (2016) 326-338. 
[17] D. Bates, Post-87 Crash fears in S\&P 500 Futures option market, J. Econometrices 94 (2000) 181-238.

[18] B. Eraker, Do stock prices and volatility jump? Reconciling evidence from spot and option prices, J. Finance 59 (2004) 1367-1403.

[19] W.L. Xiao, W.G. Zhang, X.L. Zhang, X.Y. Chen, The valuation of equity warrants under the fractional Vasicek process of the short-term interest rate, Physica A. 394 (2014) 320-337.

[20] I.E. Ouadghiri, R. Uctum, Jumps in equilibrium prices and asymmetric news in foreign exchange markets, Econ. Model. 54 (2016) 218-234.

[21] M. Broadie, O. Kaya, Exact simulation of stochastic volatility and other affine jump diffusion processes, Oper. Res. 2 (2006) $217-231$.

[22] A. Kaeck, C. Alexander, Stochastic volatility jump-diffusions for European equity index dynamics, Eur. Financ. Manag. 19 (3) (2013) 470-496.

[23] V. Todorov, Econometric analysis of jump-driven stochastic volatility models, J. Econometrics, 160 (2011) 12-21.

[24] W. Schoutens, S. Symens, The pricing of exotic options by Monte-Carlo simulations in a Levy market with stochastic volatility, Int. J. Theor. Appl. Finance 6 (2003) 839-864.

[25] S. M. Zhang, L.H. Wang, Fast Fourier transform option pricing with stochastic interest rate, stochastic volatility and double jumps, Appl. Math. Comput. 219 (2013) 10928-10933.

[26] S. M. Zhang, L.H. Wang, A fast numerical approach to option pricing with stochastic interest rate, stochastic volatility and double jumps, Commun. Nonlinear Sci. Numer. Simulat. 18 (2013) 1832-1839.

[27] J.X. Huang, W.L. Zhu, X.F. Ruan, Option pricing using the fast Fourier transform under the double exponential jump model with stochastic volatility and stochastic intensity, J. Comput. Appl. Mathe. 263 (2014) 152-159.

[28] P. Carr, D. Madan, Option valuation using the fast Fourier transform, J Comput Finance. 2 (2001) 61-73.

[29] R. Lord, C. Kahl, Optimal Fourier inversion in semi-analytical option pricing, J Comput Finance. 10 (2007) 1-30.

[30] C. Menn, S.T. Rachev, Calibrated FFT-based density approximations for $\alpha$-stable distributions, Comput. Stat. Data Anal. 50 (8) (2006) 1891-1904.

[31] M. Scherer, S.T. Rachev, Y.S. Kim, F.J. Fabozzi, Approximation of skewed and leptokurtic return distributions, Appl. Financ. Econ. 22 (16) (2012) 1305-1316.

[32] X.L. Gong, X.T. Zhuang, American option valuation under time changed tempered stable Lévy processes, Phys. A 466 (2017) $57-68$. 